\title{
Übersichten
}

Herzschr Elektrophys $2021 \cdot 32: 257-263$ https://doi.org/10.1007/s00399-021-00749-4 Eingegangen: 22. Februar 2021

Angenommen: 2. März 2021

Online publiziert: 30. März 2021

c c Der/die Autor(en) 2021

Ruben Schleberger · Andreas Rillig · Paulus Kirchhof · Andreas Metzner · \section{Bruno Reissmann}

Klinik und Poliklinik für Kardiologie, Universitäres Herz- und Gefäßzentrum UKE Hamburg, Universitätsklinikum Hamburg-Eppendorf, Hamburg, Deutschland

\section{Update Vorhofflimmern: Die ESC- Leitlinien 2020 sowie aktuelle Daten zur frühen antiarrhythmischen Therapie}

le Antikoagulation zur Verhinderung von Schlaganfällen sowie die Erkennung und Behandlung kardiovaskulärer Begleiterkrankungen. Zudem wird üblicherweise eine frequenzregulierende Therapie eingeleitet [3]. Dennoch treten auch unter genannten Maßnahmen weiterhin bei ca. $5 \%$ der Patienten pro Jahr kardiovaskuläre Komplikationen auf [4, 5].

\section{Die aktuellen ESC-Leitlinien}

Die aktuellen Vorhofflimmer-Leitlinien der European Society of Cardiology (ESC) sind im August 2020 im Rahmen des ESC Online-Kongresses präsentiert worden [6]. Der Behandlungspfad „CC to $\mathrm{ABC}^{\text {" }}$ (CC: Confirm and characterise, $\mathrm{ABC}$ : Avoid stroke/anticoagulation; better symptom control; cardiovascular risk factors and concomitant diseases) zieht sich als roter Faden durch die Leitlinien, umfasst wesentliche Aspekte der Morbidität geführt haben, sind die ora-

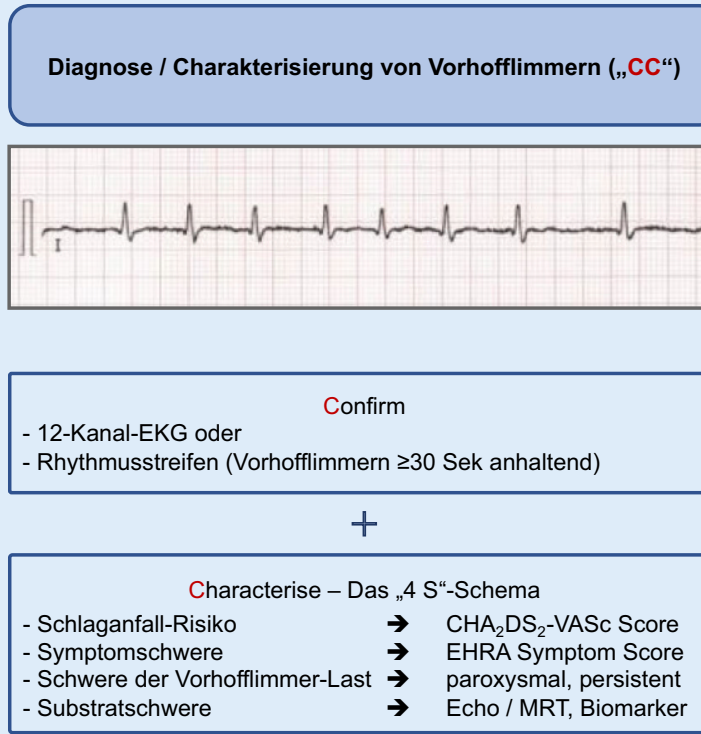

Therapieschema (,ABC“)

Avoid Stroke

Niedrigrisiko-Patienten erkennen: $\mathrm{CHA}_{2} \mathrm{DS}_{2}$-VASc $0(\mathrm{~m}) / 1(\mathrm{w})$

- Antikoagulation anbieten: $\mathrm{CHA}_{2} \mathrm{DS}_{2}-\mathrm{VASc} \geq 1(\mathrm{~m}) / \geq 2(\mathrm{w})$

- Blutungsrisiko evaluieren (HAS-BLED Score): Risikoreduktion

- Medikament auswählen: NOAK oder Marcumar (TTR >70\%)

$+$

Better Symptom Control

- Frequenzkontrolle optimieren

- Rhythmuskontrolle erwägen: Antiarrhythmika, Katheterablation

\section{$+$}

Comorbidities

- Komorbiditäten therapieren: Arterielle Hypertonie, Diabetes,

Obstruktives Schlafapnoesyndrom etc.

- Lebensstil modifizieren / Risikofaktoren reduzieren:

Gewichtsverlust, körperliches Training, Reduktion von Alkohol-

und Nikotinkonsum

Abb. $1 \triangle$ "CC to ABC" Diagnose- und Therapiealgorithmus, der in den Leitlinien empfohlen wird. Echo Echokardiographie, EKG Elektrokardiogramm, $m$ männlich, MRT Magnetresonanztomographie, NOAK Neue orale Antikoagulanzien, TTR "time in therapeutic range", $w$ weiblich. (Mod. nach [6]) 


\begin{tabular}{|l|l|l|}
\hline Empfehlungsgrad & Definition & Formulierung \\
\hline Klasse I & $\begin{array}{l}\text { Evidenz und/oder allgemeine Übereinkunft, dass eine Therapieform oder } \\
\text { diagnostischen Maßnahme vorteilhaft, nützlich oder wirksam ist. }\end{array}$ & $\begin{array}{l}\text { Wird empfohlen oder ist } \\
\text { indiziert }\end{array}$ \\
\hline Klasse II & $\begin{array}{l}\text { Widersprüchliche Evidenz und/oder unterschiedliche Meinungen über den } \\
\text { Nutzen/die Effektivität einer Therapieform oder diagnostischen Maßnahme. }\end{array}$ & \\
\hline Klasse Ila & $\begin{array}{l}\text { Evidenz/Meinungen favorisieren den Nutzen bzw. die Effektivität einer } \\
\text { Maßnahme. }\end{array}$ & Sollte erwogen werden \\
\hline Klasse IIb & $\begin{array}{l}\text { Der Nutzen/die Effektivität einer Maßnahme ist weniger gut durch } \\
\text { Evidenz/Meinungen belegt. }\end{array}$ & Kann erwogen werden \\
\hline Klasse III & $\begin{array}{l}\text { Evidenz und/oder allgemeine Übereinkunft, dass eine Therapieform oder } \\
\text { diagnostische Maßnahme nicht effektiv, nützlich oder heilsam ist und im } \\
\text { Einzelfall schädlich sein kann. }\end{array}$ & Wird nicht empfohlen \\
\hline
\end{tabular}

Abb. $2 \Delta$ Empfehlungs- und Evidenzgrade. (Nach [6])

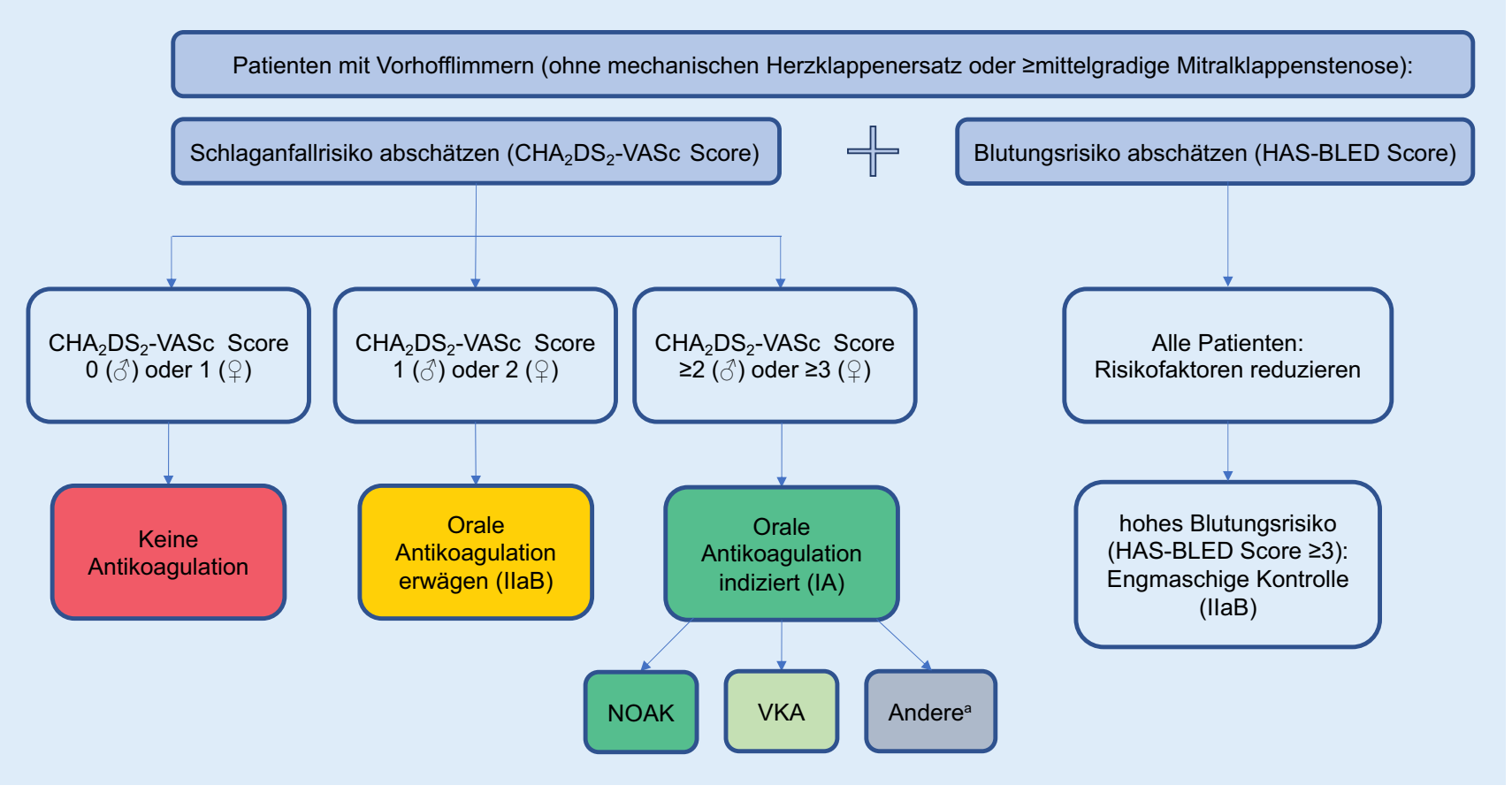

Abb. 3 A Flussdiagramm der Entscheidungsfindung zur oralen Antikoagulation. NOAK neue orale Antikoagulanzien, VKA Vitamin-K-Antagonisten, 'keine orale Antikoagulation oder Verschluss des linksatrialen Appendage im Fall klarer Kontraindikationen für orale Antikoagulation. (Mod. nach [6])

Therapie des Vorhofflimmerns und kann Behandelnden als wichtiges Instrument dienen (• Abb. 1).

\section{Screening}

Die Notwendigkeit der frühen Diagnose des Vorhofflimmerns wird durch die hohe Zahl asymptomatischer Patienten sowie den relativ hohen Anteil ischämi- scher Schlaganfälle, die auf Vorhofflimmern zurückzuführen sind, begründet. Grundsätzlich empfehlen die Leitlinien wie auch in den Vorjahren ein kosteneffizientes, opportunistisches Screening bei allen Patienten > 65 Jahre (IB; Empfehlungs- und Evidenzgrade siehe - Abb. 2). Strukturierte Empfehlungen bezüglich des weiteren Managements sollten vorgehalten werden (IB). Auf- gewertet wurde die Empfehlung, für Patienten mit höherem Risikoprofil, wie z. B. Alter $>75$ Jahre, ein systematisches Screening zu erwägen (IIaB).

\section{"CC - confirm and characterise"}

Zur Diagnosestellung von Vorhofflimmern wird in den neuen Leitlinien ein 12-Kanal-EKG oder 1-/3-Kanal-EKG mit 
über 30 Sekunden anhaltendem Vorhofflimmern gefordert (IB).

Der neue Klassifizierungsalgorithmus "4S-AF Schema“ soll die wichtigsten Aspekte des Vorhofflimmerns nach Diagnosestellung strukturiert analysieren (IIaC). Die vier „S“ stehen für „stroke risk, symptom severity, severity of $\mathrm{AF}$ burden“ und „substrate severity“. Unter den Schlagworten verbergen sich die bekannten klinischen Tools $\mathrm{CHA}_{2} \mathrm{DS}_{2}$ VASc-Score, EHRA-Symptom-Score sowie das zeitliche Muster der Vorhofflimmerepisoden (paroxysmal, persistierend, langanhaltend persistierend und permanent). Die Evaluation der „substrate severity" umfasst die Nutzung diagnostischer Mittel (Echokardiographie/MRT/ Biomarker) und Erfassung der Komorbiditäten.

\section{Therapie von Vorhofflimmern}

Im Zentrum der Leitlinien 2020 steht ein integratives Behandlungskonzept im Sinne einer interdisziplinären Betreuung durch Hausärztin/Hausarzt, Kardiologin/Kardiologe, Klinikteam, Apotheker/in u. a. (IIaB).

\section{"A - anticoagulation/avoid stroke“}

Der $\mathrm{CHADS}_{2} \mathrm{DS}_{2}$-VASc-Score als Kompromiss aus Praktikabilität und Genauigkeit ist maßgebend (IA; - Abb. 3; - Tab. 1). Das weibliche Geschlecht wird weiter im Score erfasst, da es sich um einen altersabhängigen Risikomodifikator handelt. Bei Vorliegen eines Risikofaktors für thrombembolische Ereignisse (ohne weibliches Geschlecht) sollte der Beginn einer oralen Antikoagulation erwogen werden (IIaB). Bei Patienten mit $\mathrm{CHADS}_{2} \mathrm{DS}_{2}$-VASc-Score 2 (Männer) bzw. 3 (Frauen) sollte eine orale Antikoagulation definitiv erfolgen (IA). Bei initial niedrigem Schlaganfallrisiko ist nach vier bis sechs Monaten eine Reevaluation zu empfehlen, da Risikofaktoren häufig erst nach Erstdiagnose von Vorhofflimmern zu Tage treten (IIaB).

Mit Beginn der oralen Antikoagulation sollte bei jedem Patienten auch das Blutungsrisiko evaluiert werden (IB). Bei hohem Blutungsrisiko (HAS-BLEDScore $>2$ ) werden anstatt prophylakti-

Herzschr Elektrophys 2021 · 32:257-263 https://doi.org/10.1007/s00399-021-00749-4

(c) Der/die Autor(en) 2021

R. Schleberger · A. Rillig · P. Kirchhof · A. Metzner · B. Reissmann

\section{Update Vorhofflimmern: Die ESC-Leitlinien 2020 sowie aktuelle Daten zur frühen antiarrhythmischen Therapie}

\section{Zusammenfassung}

Vorhofflimmern geht mit einer beträchtlichen Belastung für Patienten und das Gesundheitssystem einher. Jeder dritte heute 55-Jährige wird zeitlebens an Vorhofflimmern erkranken. Trotz Verbesserungen des Managements von Vorhofflimmern verbleibt ein erhöhtes Risiko für kardiovaskuläre Ereignisse. Die neuen Vorhofflimmer-Leitlinien der European Society of Cardiology stellen ein integratives Therapiekonzept in den Mittelpunkt. Der neue Therapie- und Diagnosealgorithmus "CC to $A B C$ " umfasst sowohl Diagnosestellung ("confirm") und Klassifizierung ("characterise") als auch Therapie („avoid stroke", "better symptom control", „comorbidities"). Neue orale Antikoagulanzien stehen unter Anwendung des $\mathrm{CHA}_{2} \mathrm{DS}_{2}$-VASC-Scores im Zentrum der Vorbeugung von Schlaganfällen. Neben der Frequenzregulierung werden rhythmuserhaltende Maßnahmen wie die medikamentöse antiarrhythmische Therapie oder die Katheterablation mit prognostischer Relevanz für bestimmten Patientengruppen empfohlen. Die Adressierung von Risikofaktoren und Komorbiditäten wie arterielle Hypertonie, Diabetes mellitus, Adipositas und Schlafapnoe wirkt ergänzend und sollte Teil jedes Behandlungskonzepts sein. Die im August 2020 publizierte EAST-AFNET 4Studie zeigt als erste große randomisierte Studie, dass die frühe rhythmuserhaltende Therapie zusätzlich zur leitlinienbasierten Vorhofflimmertherapie zur Vermeidung von kardiovaskulärem Tod und Schlaganfällen beiträgt. In Anbetracht der Sicherheit von Antiarrhythmika und Katheterablation sollte die frühe Einleitung einer rhythmuserhaltenden Therapie bei allen Patienten in den ersten Monaten nach der Erstdiagnose von Vorhofflimmern erwogen werden, um positive Effekte nicht zu verpassen.

\section{Schlüsselwörter}

Atriale Tachyarrhythmien - Therapiealgorithmus · Medikamentöse antiarrhythmische Therapie · Katheterablation · EAST-AFNET 4

\section{Update atrial fibrillation: the 2020 ESC guidelines and recent data on early rhythm control}

\section{Abstract}

Atrial fibrillation (AF) can be a significant burden for patients as well as the health care system. Every third 55-year-old will develop AF. Despite improvements of disease management, a significant risk for cardiovascular events remains. The current AF guidelines of the European Society of Cardiology focus on an integrative therapy approach. The new algorithm " $\mathrm{CC}$ to $A B C$ " comprises recommendations for diagnosis ("confirm" and "characterise") and treatment ("avoid stroke", "better symptom control", "comorbidities") of AF. Direct oral anticoagulants administered according to the $\mathrm{CHA}_{2} \mathrm{DS}_{2}$-VASc score remain the corner stones of stroke prevention. Besides the concept of heart rate control, rhythm control therapy like antiarrhythmic drugs or catheter ablation is recommended to relieve symptoms and in certain patient groups for the improvement of prognosis. Therapy of comorbidities and reduction of risk factors like hypertension, diabetes mellitus, obesity and obstructive sleep apnoea should be part of any comprehensive treatment approach. The results of the randomized, prospective EAST-AFNET 4 trial were published in August 2020. The trial shows that an early rhythm control therapy can lead to a reduction of cardiovascular mortality and incidence of stroke additionally to guideline-based AF management. Given the safety profile and potential positive effects of antiarrhythmic drugs and catheter ablation, early initiation of rhythm control therapy should be considered in every patient during the first months after diagnosis of AF.

\section{Keywords}

Atrial tachyarrhythmias - Therapy algorithm . Antiarrhythmic drug treatment - Catheter ablation - EAST-AFNET 4 


\begin{tabular}{|c|c|}
\hline $\begin{array}{l}\mathrm{CHA}_{2} \mathrm{DS}_{2} \text {-VASc-Score Risikofakto- } \\
\text { ren }\end{array}$ & Punkte \\
\hline $\begin{array}{l}\text { Herzinsuffizienz } \\
\text { Zeichen/Symptome der Herzinsuf- } \\
\text { fizienz oder objektive Hinweise auf } \\
\text { eine reduzierte linksventrikuläre } \\
\text { Ejektionsfraktion }\end{array}$ & +1 \\
\hline $\begin{array}{l}\text { Hypertension } \\
\text { Blutdruck in Ruhe }>140 / 90 \mathrm{~mm} \mathrm{Hg} \\
\text { in } 2 \text { Messungen oder antihyperten- } \\
\text { sive Therapie }\end{array}$ & +1 \\
\hline Alter $\geq 75$ Jahre & +2 \\
\hline $\begin{array}{l}\text { Diabetes mellitus } \\
\text { Nüchtern-Glukose }>125 \mathrm{mg} / \mathrm{dl} \\
\text { ( } 7 \mathrm{mmol} / \mathrm{l}) \text { oder Behandlung mit } \\
\text { Antidiabetika/Insulin }\end{array}$ & +1 \\
\hline $\begin{array}{l}\text { Früherer Schlaganfall, TIA, Thromb- } \\
\text { embolie }\end{array}$ & +2 \\
\hline $\begin{array}{l}\text { Vaskuläre Erkrankung } \\
\text { Z.n. Myokardinfarkt, PAVK oder } \\
\text { Verkalkung der Aorta }\end{array}$ & +1 \\
\hline Alter 65-74 Jahre & +1 \\
\hline Geschlecht (weiblich) & +1 \\
\hline
\end{tabular}

schem Verzicht auf eine indizierte orale Antikoagulation eine Adressierung modifizierbarer Risikofaktoren und eine engmaschige klinische Überwachung empfohlen.

Bei Auswahl der Medikation zur oralen Antikoagulation zählen die neuen oralen Antikoagulanzien (NOAKs) wie gehabt aufgrund ihrer Effektivität, ihres Sicherheitsprofils sowie der einfachen Anwendung als Medikamente der ersten Wahl (IA). Im Fall einer Therapie mit Vitamin-K-Antagonisten sollte eine „time in therapeutic range" $>70 \%$ beiZiel INR (,international normalized ratio") von 2,0-3,0 angestrebt werden (IB). Eine Dosisreduktion des jeweiligen NOAK abseits der evidenzbasierten Empfehlungen sollte vermieden werden.

\section{„B - better symptom control“}

Die beiden Hauptkonzepte der Vorhofflimmertherapie bleiben weiterhin die frequenzregulierende und die rhythmuserhaltende Therapie. Erstere kann hierbei als Basistherapie betrachtet werden. Keine Änderung ergibt sich beim anzustrebenden Frequenzziel mit $<110$ Schlägen/min (IIaB). Die Empfehlung beruht u. a. auf Daten der RACE-, RACE II- so- wie AFFIRM-Studie, wobei der Zielwert kontrovers diskutiert wird [7-9].

Die langfristige rhythmuserhaltende Therapie wird von den Autoren als symptomorientierte Therapie eingestuft und ist in der Regel Patienten mit symptomatischem Vorhofflimmern vorbehalten. Zur initialen Entscheidungsfindung bezüglich der Art der antiarrhythmischen Therapie (medikamentös vs. interventionell) steht in den Leitlinien 2020 der Patientenwunsch im Vordergrund. Die Katheterablation ist grundsätzlich sicher und der medikamentösen antiarrhythmischen Therapie bezüglich Symptomkontrolle und Erhalt des Sinusrhythmus überlegen [10-12].

Als Erstlinientherapie ist die Katheterablation bei Patienten mit paroxysmalem Vorhofflimmern eine IIaB-Empfehlung. Neu ist eine IIbC-Empfehlung der Erstlinientherapie für Patienten mit persistierendem Vorhofflimmern ohne schwerwiegende Risikofaktoren für Rezidive.

Flecainid und Propafenon sollten nur bei Patienten ohne strukturelle Herzerkrankung eingesetzt werden (IA). Amiodaron ist das Medikament mit der höchsten Effektivität und kann auch bei Patienten mit struktureller Herzerkrankung und akuter Herzinsuffizienz eingesetzt werden [13]. Engmaschige klinische sowie laborchemische Überwachungen sind aufgrund der häufigen Nebenwirkungen der Amiodarontherapie notwendig, sodass wenn möglich anderen Antiarrhythmika der Vorzug gegeben werden sollte (IA). Dronedaron hat ein besseres Sicherheitsprofil als Amiodaron, jedoch auch eine niedrigere Effektivität [14, 15]. Der Einsatz wird als Langzeittherapie bei Patienten mit erhaltener oder leicht reduzierter (stabiler) linksventrikulärer Ejektionsfraktion empfohlen (IA). Im Unterschied zu einer Therapie mit Flecainid oder Propafenon schließt dies Patienten mit koronarer und hypertensiver Herzerkrankung ein. Bei allen Antiarrhythmika werden EKGKontrollen zu Beginn der Therapie sowie nach ein bis zwei Wochen (Flecainid/ Propafenon) bzw. vier Wochen (Amiodaron/Dronedaron) empfohlen.

Für ausgewählte Patientenkollektive, wie z.B. Patienten mit eingeschränkter linksventrikulärer Ejektionsfraktion, konnte eine Verbesserung der Mortalität durch die Katheterablation gezeigt werden (CASTLE-AF-Studie; [16]). Auf Grundlage dieser aktuellen Daten wird eine Klasse-IIaB-Empfehlung für die symptomunabhängige Katheterablation bei Patienten mit Herzinsuffizienz und reduzierter linksventrikulärer Ejektionsfraktion abgegeben. Weiterhin gibt es nun eine Empfehlung IB der Katheterablation für Patienten mit hochgradigem Verdacht auf Tachymyopathie.

Als Zweitlinientherapie nach frustraner bzw. nichttolerierter medikamentöser antiarrhythmischer Therapie wird die Katheterablation sowohl für Patienten mit paroxysmalem als auch mit persistierendem Vorhofflimmern empfohlen (IA).

Die orale Antikoagulation sollte nach Katheterablation bei allen Patienten für mindestens zwei Monate und im Anschluss nach $\mathrm{CHA}_{2} \mathrm{DS}_{2}$-VASc-Score fortgeführt werden (IC).

\section{"C - cardiovascular risk factors and concomitant diseases"}

Das Auftreten von Vorhofflimmern wird u. a. durch den Lebensstil, kardiovaskuläre Risikofaktoren und kardiale Grunderkrankungen beeinflusst. Die RACE IIIStudie zeigte, dass die gezielte Behandlung von Risikofaktoren und Grunderkrankungen bei Patienten mit Herzinsuffizienz zu einer Stabilisierung des Sinusrhythmus führt [17]; diese wird daher als Teil der Behandlung des Vorhofflimmerns empfohlen (IB).

\section{Frühe antiarrhythmische Therapie - aktuelle Studien- daten}

Die Ergebnisse der ebenfalls im Rahmen des ESC-Kongresses 2020 vorgestellten EAST-AFNET 4-Studie („,early treatment of atrial fibrillation for stroke prevention trial“; - Tab. 2) fordern möglicherweise schon ein erstes fokussiertes Update der Leitlinie [4].

Die Studie prüfte die Hypothese, ob eine früh begonnene rhythmuserhaltende Therapie das Auftreten von kardiovaskulären Komorbiditäten und kardiovaskulären Ereignissen verhindern kann. Insgesamt 2789 Patienten 
Tab. 2 Aktuelle Daten zur frühen antiarrhythmischen Therapie

\begin{tabular}{ll} 
Studie (Jahr) & Studienpopulation \\
\hline EAST-AFNET 4 & Patienten mit kürzlich dia- \\
(2020) [4] & $\begin{array}{l}\text { gnostiziertem Vorhofflimmern } \\
\text { (Erstdiagnose } \leq 1 \text { Jahr vor Stu- } \\
\text { dieneinschluss; } n=2789)\end{array}$ \\
& $\begin{array}{l}\text { Risikofaktoren für thromb- } \\
\text { embolische Ereignisse (Alter } \\
>75 \text { Jahre, Z.n. TIA/Schlaganfall } \\
\text { oder } \geq 2 \text { der folgenden Kriteri- } \\
\text { en: Alter }>65 \text { Jahre, weibliches } \\
\text { Geschlecht, Herzinsuffizienz, } \\
\text { arterieller Hypertonus, Dia- } \\
\text { betes mellitus, schwere KHK, } \\
\text { chronische Niereninsuffizienz } \\
\text { (Stadium III/IV), LV-Septumdi- } \\
\text { cke }>15 \text { mm) }\end{array}$ \\
\hline
\end{tabular}

EARLY-AF

(2021) [12]

\section{Patienten mit kürzlich dia-} gnostiziertem (Erstdiagnose $\leq 2$ Jahre), therapienaivem (keine regelhafte $A A D$-Einnahme), symptomatischem, paroxysmalem Vorhofflimmern $(n=303)$
STOP-AF First Patienten mit therapienaivem (2021) [18] (AAD <7 Tage), symptomatischem, paroxysmalem Vorhofflimmern $(n=203)$
Behandlungsgruppen

Frühe rhythmuserhaltende

Therapie: PVI und/oder AAD.

EKV nach Notwendigkeit

Kontrollgruppe: Frequenz-

kontrolle, rhythmuserhaltende Therapie bei persistieren-

den Symptomen

Fortführung von OAK nach

$\mathrm{CHA}_{2} \mathrm{DS}_{2}$-VASc-Score in bei-

den Gruppen

Interventionsgruppe: PVI mittels Kryoballon

Kontrollgruppe: AAD nach

Leitlinie (Auftitration wäh-

rend 90 Tage Blanking-Peri-

ode)

Implantation eines Ereignis-

rekorders in beiden Gruppen

\section{Endpunkte}

Primärer Endpunkt: kardiovaskulärer

Tod, Schlaganfall, Hospitalisierung aufgrund von Herzinsuffizienz oder akutem Koronarsyndrom

Primärer Sicherheitsendpunkt: Tod,

Schlaganfall, Auswahl von SAEs

Mediane Dauer des Follow-ups:

5,1 Jahre
Ergebnisse

249 (3,9 pro 100 Personenjahre) Ereignisse des pri-

mären Endpunkts (rhythmuserhaltende Therapie) vs. 316 (5,0 pro 100 Personenjahre) Ereignisse (Kontrollgruppe); HR 0,79; $96 \%$ KI [0,66-0,94]; $p=0,005$

Primärer Sicherheitsendpunkt: 231 (16,6\%) Patienten mit rhythmuserhaltender Therapie vs. 223 (16,0\%) Patienten der Kontrollgruppe; $p<0,001$

Primärer Endpunkt: erstes Rezidiv einer Primärer Endpunkt: 66 atrialen Tachyarrhythmie (>30 s) ab Ende der Blanking-Periode (90 Tage post interventionem)

Sekundäre Endpunkte (Auswahl): symptomatische Rezidive; Vorhofflimmerlast; SAE

Follow-up-Dauer: 12 Monate

(42,9\% der Patienten der Interventionsgruppe) vs. 101 (67,8\% der Patienten der Kontrollgruppe); HR 0,48; $95 \%$ KI $[0,35-0,66] ; p<0,001$ Symptomatische Rezidive: 17 $(11,0 \%)$ vs. 39 (26,2\%); HR $0,39[0,22-0,68]$

Vorhofflimmerlast (Median): $0 \%$; IQR $(0-0,08)$ vs. $0,13 \%$; IQR $(0-1,60)$

SAE: 5 (3,2\%) vs. 6 (4,0\%); RR 0,$81 ; 95 \%$ KI $[0,25-2,59]$

Interventionsgruppe: PVI mittels Kryoballon

Kontrollgruppe: AAD nach Leitlinie (Auftitration während 90 Tage Blanking-Periode)
Primärer Endpunkt: Behandlungserfolg, definiert als Freiheit von Rezidiv einer atrialen Tachyarrhythmie ( $>30 \mathrm{~s}$ ) ab Ende der Blanking-Periode ( 90 Tage post interventionem), linksatriale Reablation, frustrane primäre Ablation, Kardioversion, Nutzung von AAD nach Blanking-Periode (nur Interventionsgruppe)

Primärer Sicherheitsendpunkt (nur Interventionsgruppe): Perikarderguss innerhalb 30 Tagen post interventionem, Pulmonalvenenstenose, ösophagoatriale Fistel, permanente Phrenikusparese, TIA/Schlaganfall, Myokardinfarkt, schwere Gefäßkomplikation, schwere Blutung (innerhalb von 7 Tagen postprozedural)

Follow-up-Dauer: 12 Monate
Primärer Endpunkt (Behandlungserfolg; Kaplan-Meier-

Schätzer): 74,6\%; $95 \% \mathrm{KI}$ [65,0-82,0] (Interventionsgruppe) vs. $45,0 \% ; 95 \% \mathrm{KI}$ $[34,6-54,7]$ (Kontrollgruppe); $p<0,001$

Primärer Sicherheitsendpunkt (Kaplan-Meier-Schätzer): 1,9\%; $95 \%$ KI [0,5-7,5] SAE: 15 (14\%) vs. 14 (14\%); ns

$A A D$ antiarrhythmische medikamentöse Therapie, EKV Kardioversion, $L V$ linker Ventrikel, HR Hazard-Ratio, IQR „interquartile range", $K I$ Konfidenzintervall, KHK koronare Herzerkrankung, PVI Pulmonalvenenisolation, RR Risikoreduktion, SAE "serious adverse event", TIA transitorische ischämische Attacke

mit Vorhofflimmern wurden im Median 36 Tage nach Diagnosestellung in 135 Zentren randomisiert. In der Interventionsgruppe erfolgte eine frühe medikamentöse oder interventionelle rhythmuserhaltende Therapie. In der Kontrollgruppe erfolgte die Therapie nach den gültigen Leitlinien, d.h. zunächst Frequenzregulierung und Hinzufügen einer rhythmusstabilisierenden Therapie bei weiterbestehenden Symptomen. Das mediane Follow-up betrug 5,1 Jahre. Ein Ereignis des zusammengesetzten primären Endpunkts (kardiovaskulärer Tod, Schlaganfall, Hospitalisierung mit Herzinsuffizienz oder akutem Koronarsyndrom) trat bei 249 Patienten mit früher rhythmuserhaltender Therapie und bei 316 Patienten mit konventioneller Behandlung auf (Hazard-Ratio 0,79;
$96 \%$ Konfidenzintervall $[0,66-0,94]$; $p=0,005)$. Die Einzelkomponenten des kombinierten Endpunkts traten in der Gruppe mit früher rhythmuserhaltender Therapie ebenfalls vermindert auf. Die Gesamtmortalität war statistisch nicht unterschiedlich $(9,9 \%$ bei Patienten mit früher rhythmuserhaltender Therapie, $11,8 \%$ bei Patienten der Kontrollgruppe). „Serious adverse events related to 
rhythm control“ waren insgesamt sehr selten. So wiesen nur 28/1395 Patienten mit früher rhythmuserhaltender Therapie $(0,28 \% / \mathrm{Jahr})$ und $9 / 1394 \mathrm{~Pa}$ tienten in der Kontrollgruppe $(0,13 \% /$ Jahr) schwerwiegende unerwünschte Ereignisse wegen antiarrhythmischer Therapie auf. Ablationskomplikationen fanden sich bei 9/1394 Patienten mit früher rhythmuserhaltender Behandlung $(0,13 \% / \mathrm{Jahr})$ und 2/1394 Patienten in "usual care“ $(0,03 \% / \mathrm{Jahr} ; p<0,001)$.

Die EAST-AFNET 4-Studie zeigte als erste große randomisiert-kontrollierte Studie, dass die frühe rhythmuserhaltende Therapie zur Vermeidung von kardiovaskulärem Tod und Schlaganfällen wesentlich beiträgt. Für Patienten mit neu aufgetretenem Vorhofflimmern sollte auch im Hinblick auf die erst kürzlich erschienenen Leitlinien das Therapiekonzept hin $\mathrm{zu}$ einer frühen rhythmuserhaltenden Therapie angepasst werden.

Bestärkt wurden diese positiven Ergebnisse bereits Ende 2020 durch zwei nordamerikanische Studien, die ebenfalls die Relevanz der frühen Vorhofflimmertherapie untersuchten. Die EARLY-AFStudie und die STOP-AF-First-Studie zeigten beide unabhängig voneinander die Überlegenheit der interventionellen rhythmuserhaltenden Therapie mittels Kryoballonablation gegenüber einer medikamentösen antiarrhythmischen Therapie als Initialtherapie bei Patienten mit paroxysmalem Vorhofflimmern bei vergleichbarer Sicherheit (ס Tab. 2; [12, 18]).

Als Schlussfolgerung sollte in Anbetracht der Sicherheit von Antiarrhythmika und Katheterablation sowie in Kenntnis der Wirkung auf kardiovaskuläre Komplikationen eine frühe Einleitung einer rhythmuserhaltenden Therapie bei allen Patienten in den ersten Monaten nach Erstdiagnose von Vorhofflimmern erwogen werden, um positive Effekte nicht zu verpassen. Dies ist eine wesentliche Veränderung, die in ein Update der Vorhofflimmer-Leitlinien einfließen sollte.

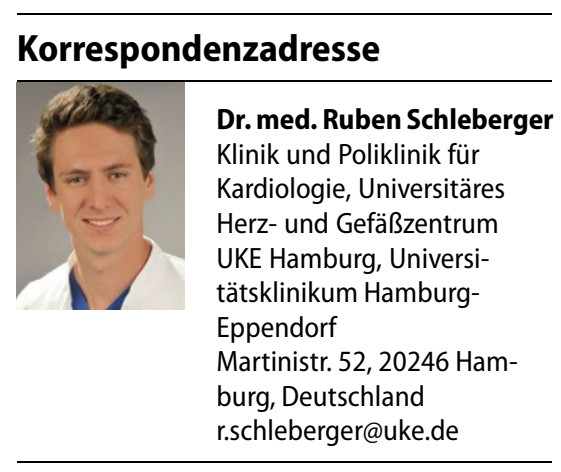

Funding. Open Access funding enabled and organized by Projekt DEAL.

\section{Einhaltung ethischer Richtlinien}

Interessenkonflikt. R. Schleberger gibt an, dass kein Interessenkonflikt besteht. A. Rillig erhielt Reisekostenzuschüsse und Vortragshonorare von Biosense Webster, Hansen Medical, Medtronic, EPSolutions und St. Jude Medical sowie Vortragshonorare von Boehringer Ingelheim und Beraterhonorare von Medtronic. P. Kirchhof wird durch European Union BigData@Heart (grant agreement EU IMI 116074), British Heart Foundation (FS/13/43/30324; PG/17/30/32961 andPG/20/22/35093;AA/18/2/34218), German Centre for Cardiovascular Research (DZHK), und die Leducq Foundation unterstützt und erhielt Unterstützung für Grundlagenforschung, translationale und klinische Forschungsprojekte von der Europäischen Union, British Heart Foundation, Leducq Foundation, Medical Research Council (UK) und vom German Centre for Cardiovascular Research sowie von mehreren Pharmaund Medizintechnikunternehmen aktiv im Bereich Vorhofflimmern und hat Honorare von mehreren derartigen Firmen in der Vergangenheit, jedoch nicht in den letzten drei Jahren erhalten und wird als Erfinder zweier Patente an der University of Birmingham (Atrial Fibrillation Therapy WO 2015140571, Markers for Atrial Fibrillation WO 2016012783) geführt. A. Metzner erhielt Vortragshonorare und Reisekostenzuschüsse von Medtronic und Biosense Webster und B. Reissmann erhielt Vortragshonorare und Reisekostenzuschüsse von Medtronic.

Für diesen Beitrag wurden von den Autoren keine Studien an Menschen oder Tieren durchgeführt. Für die aufgeführten Studien gelten die jeweils dort angegebenen ethischen Richtlinien.

Open Access. Dieser Artikel wird unter der Creative Commons Namensnennung 4.0 International Lizenz veröffentlicht, welche die Nutzung, Vervielfältigung, Bearbeitung, Verbreitung und Wiedergabe in jeglichem Medium und Format erlaubt, sofern Sie den/die ursprünglichen Autor(en) und die Quelle ordnungsgemäß nennen, einen Link zur Creative Commons Lizenz beifügen und angeben, ob Änderungen vorgenommen wurden.

Die in diesem Artikel enthaltenen Bilder und sonstiges Drittmaterial unterliegen ebenfalls der genannten Creative Commons Lizenz, sofern sich aus der Abbildungslegende nichts anderes ergibt. Sofern das betreffende Material nicht unter der genannten Creative Commons Lizenz steht und die betreffende Handlung nicht nach gesetzlichen Vorschriften erlaubt ist, ist für die oben aufgeführten Weiterverwendungen des Materials die Einwilligung des jeweiligen Rechteinhabers einzuholen.

Weitere Details zur Lizenz entnehmen Sie bitte der Lizenzinformation auf http://creativecommons.org/ licenses/by/4.0/deed.de.

\section{Literatur}

1. Staerk L, Wang B, Preis SR, Larson MG, Lubitz SA, Ellinor PT et al (2018) Lifetime risk of atrial fibrillation according to optimal, borderline, or elevated levels of risk factors: cohort study based on longitudinal data from the Framingham Heart Study. BMJ 361:k1453

2. Fabritz L, Crijns HJGM, Guasch E, Goette A, Haeusler KG, Kotecha D et al (2021) Dynamic risk assessment to improve quality of care in patients with atrial fibrillation: the 7th AFNET/EHRA Consensus Conference. EP Eur. https://doi.org/10. 1093/europace/euaa279

3. KotechaD, Bunting KV, Gill SK, MehtaS, StanburyM, Jones JC et al (2020) Rate control therapy evaluation in permanent atrial fibrillation (RATEAF) team. Effect of Digoxin vs bisoprolol for heart rate control in atrial fibrillation on patient-reported quality of life: the RATE-AF randomized clinical trial. JAMA 324:2497-2508

4. KirchhofP,CammAJ,Goette A, Brandes A, EckardtL, Elvan A et al (2020) Early rhythm-control therapy in patients with atrial fibrillation. N Engl J Med 383:1305-1316

5. Marijon E, Le Heuzey J-Y, Connolly S, Yang S, Pogue J, Brueckmann M et al (2013) Causes of death and influencing factors in patients with atrial fibrillation: a competing-risk analysis from the randomized evaluation of long-term anticoagulant therapy study. Circulation 128:2192-2201

6. Hindricks G, Potpara T, Dagres N, Arbelo E, Bax JJ, Blomström-Lundqvist Cetal (2020) ESC Guidelines for the diagnosis and management of atrial fibrillation developed in collaboration with the European Association of Cardio-Thoracic Surgery (EACTS). Eur Heart J 42:373-498

7. Wyse DG, Waldo AL, DiMarco JP, Domanski MJ, Rosenberg Y, Schron EB et al (2002) Atrial Fibrillation Follow-up Investigation of Rhythm Management (AFFIRM) Investigators. A comparison of rate control and rhythm control in patients with atrial fibrillation. NEngl J Med 347:1825-1833

8. Van Gelder IC, Hagens VE, Bosker HA, Kingma JH, Kamp O, Kingma T et al (2002) Rate Control versus Electrical Cardioversion for Persistent Atrial Fibrillation Study Group. A comparison of rate control and rhythm control in patients with recurrent persistent atrial fibrillation. $N$ Engl J Med 347:1834-1840

9. Van Gelder IC, Groenveld HF, Crijns HJGM, Tuininga YS, Tijssen JGP, Alings AM et al (2010) Lenient versus strict rate control in patients with atrial fibrillation. NEngl J Med 362:1363-1373

10. Packer DL, Mark DB, Robb RA, Monahan KH, Bahnson TD, Poole JE et al (2019) Effect of catheter ablation vs antiarrhythmic drug therapy on mortality, stroke, bleeding, and cardiac arrest among patients with atrial fibrillation: the CABANA randomized clinical trial. JAMA 321:1261-1274

11. Blomström-Lundqvist C, Gizurarson S, Schwieler J, Jensen SM, Bergfeldt L, Kennebäck G et al (2019) Effect of catheter ablation vs antiarrhythmic medication on quality of life in patients with atrial 
fibrillation: the CAPTAF randomized clinical trial. JAMA 321:1059-1068

12. Andrade JG, Wells GA, Deyell MW, Bennett M, Essebag V, Champagne J et al (2021) Cryoablation or drug therapy for initial treatment of atrial fibrillation. N Engl J Med 384:305-315

13. Freemantle $N$, Lafuente-Lafuente $C$, Mitchell $S$, Eckert L, Reynolds M (2011) Mixed treatment comparison of dronedarone, amiodarone, sotalol, flecainide, and propafenone, for the management of atrial fibrillation. Europace 13:329-345

14. Piccini JP, Hasselblad V, Peterson ED, Washam JB, Califf RM, Kong DF (2009) Comparative efficacy of dronedaroneandamiodarone for the maintenance of sinus rhythm in patients with atrial fibrillation. J Am Coll Cardiol 54:1089-1095

15. Gao S, Dai W, Zhang L, Juhaeri J, Wang Y, Caubel P (2013) Risk of cardiovascular events, stroke, congestive heart failure, interstitial lung disease, and acute liver injury: dronedarone versus amiodarone and other Antiarrhythmics. J Atr Fibrillation 6:890

16. Marrouche NF, Brachmann J, Andresen D, Siebels J, Boersma L, Jordaens Letal (2018) Catheter ablation for atrial fibrillation with heart failure. N Engl J Med 378:417-427

17. Rienstra $M$, Hobbelt $A H$, Alings $M$, Tijssen JGP, Smit MD, Brügemann J et al (2018) Targeted therapy of underlying conditions improves sinus rhythm maintenance in patients with persistent atrial fibrillation: results of the RACE 3 trial. Eur Heart J39:2987-2996

18. Wazni OM, Dandamudi G, Sood N, Hoyt R, Tyler J, Durrani $S$ et al (2021) Cryoballoon ablation as initial therapy for atrial fibrillation. $N$ Engl $J$ Med 384(4):316-324 